\title{
Interplay between the magnetic anisotropy contributions of cobalt nanowires
}

\author{
J. Sánchez-Barriga, ${ }^{1,2, *}$ M. Lucas, ${ }^{3}$ F. Radu, ${ }^{2}$ E. Martin, ${ }^{1}$ M. Multigner, ${ }^{4,5}$ P. Marin, ${ }^{1}$ A. Hernando, ${ }^{1}$ and G. Rivero ${ }^{1}$ \\ ${ }_{1}^{1}$ Instituto de Magnetismo Aplicado (UCM-ADIF-CSIC), P.O. Box 155, 28230 Las Rozas, Madrid, Spain \\ ${ }^{2}$ Helmholtz-Zentrum Berlin für Materialien und Energie, Albert-Einstein-Strasse 15, D-12489 Berlin, Germany \\ ${ }^{3}$ Institut für Theoretische Physik, Technische Universität Berlin, Hardenbergstr. 36, D-10623 Berlin, Germany \\ ${ }^{4}$ Centro Nacional de Investigaciones Metalúrgicas (CENIM-CSIC), Avd. Gregorio del Amo 8, 28040 Madrid, Spain \\ ${ }^{5}$ Centro de Investigación Biomédica en Red en Bioingeniería, Biomateriales y Nanomedicina (CIBER-BBN), Madrid, Spain
}

(Received 17 June 2009; revised manuscript received 21 October 2009; published 23 November 2009)

\begin{abstract}
We report on the magnetic properties and the crystallographic structure of the cobalt nanowire arrays as a function of their nanoscale dimensions. X-ray diffraction measurements show the appearance of an in-plane hcp-Co phase for nanowires with $50 \mathrm{~nm}$ diameter, suggesting a partial reorientation of the magnetocrystalline anisotropy axis along the membrane plane with increasing pore diameter. No significant changes in the magnetic behavior of the nanowire system are observed with decreasing temperature, indicating that the effective magnetoelastic anisotropy does not play a dominant role in the remagnetization processes of individual nanowires. An enhancement of the total magnetic anisotropy is found at room temperature with a decreasing nanowire diameter-to-length ratio $(d / L)$, a result that is quantitatively analyzed on the basis of a simplified shape anisotropy model.
\end{abstract}

DOI: 10.1103/PhysRevB.80.184424

PACS number(s): 75.75.+a, 75.50.Tt, 75.60.Jk, 75.60.-d

The discovery of interlayer exchange coupling ${ }^{1}$ and giant magnetoresistance ${ }^{2,3}$ have promoted a tremendous advance of storage media, readout sensors, and magnetic random access memory. By further reducing the lateral size of the magnetic structures an increased performance of the devices is achieved together with a sustained interest for fundamental understanding of low-dimensional magnetism. For antiferromagnetic materials the finite-size effects lead to a scaling of the intrinsic magnetic properties such as the ordering temperature and anisotropy as well as to an enhanced contribution of the magnetically disordered surface to the macroscopic magnetization. ${ }^{4,5}$ For ferromagnetic materials with reduced dimensions, the presence of a finite magnetization leads to an increased shape anisotropy which depends strongly on the geometry of the objects. ${ }^{6}$ This manifests stronger when the lateral dimensions are touching to the nanoscale regime. Besides the enhanced shape anisotropies, the remagnetization processes such as coherent-rotation and curling modes are more favorable against the domain-wall movements. Ultimately, the manipulation of the dynamical magnetic properties of such structures in ultrashort time scales $^{7}$ needs to be complementary to an appropriate characterization and control of their magnetic and geometrical properties.

In recent years, with the aim of exhaustively tailoring and controlling properties such as perpendicular magnetic anisotropy (PMA), ${ }^{8}$ a large variety of geometries for small-sized elements is being produced by different techniques. Magnetic nanoparticles, ${ }^{9}$ nanotubes, ${ }^{10}$ micron-sized rectangular platelets and dots, ${ }^{11}$ nanowires ${ }^{12}$ or ultrathin films are typically fabricated by using various combinations of state-ofthe-art modern experimental tools. Techniques such as electron-beam lithography or imprint lithography, ${ }^{13}$ molecular-beam epitaxy or magnetron sputtering are combined to produce high-quality magnetic structures of ultrasmall sizes. ${ }^{6}$ Among them, lithography represents a top-down fabrication technique where a bulk material is reduced in size to a nanoscale pattern. Alternatively, electrodeposition ${ }^{14}$ is a very simple and inexpensive bottom-up technique which allows fabrication of large arrays of magnetic nanowires. Combined with the use of self-assembling methods for the deposition of membranes, ${ }^{17}$ it has the particular advantage of producing systems with high PMA and ultrahigh densities. In order to control the anisotropy of such a system, it is important to understand how the magnetic properties depend on the fabrication parameters and thus on the geometrical and structural properties of the nanowires. Changes in the electrodeposition parameters such as chemical composition, temperature, and $\mathrm{pH}$ of the electrolyte, ${ }^{15}$ deposition time or electrodeposition voltage ${ }^{16}$ will result in arrays of nanowires with different intrinsic magnetic properties.

We focus our study on the influences of the reduced diameter-to-length ratio $(d / L)$ on the magnetic effective anisotropies. Our main finding is that the otherwise complex demagnetization treatment of the shape anisotropy can be reduced to a much simpler expression for the coercive fields of nanoscale Co arrays, which is probed experimentally. The Co nanowires with lengths between 1 and $6 \mu \mathrm{m}$ have been electrodeposided into the pores of track-etched polycarbonate membranes with nominal pore diameters of 30 and 50 $\mathrm{nm}$. Previous to electrodeposition, the membranes were covered by a $100 \mathrm{~nm} \mathrm{Cu}$ film by sputtering technique. This acts as an electrode during the fabrication process. An agitation system was used in order to avoid hydrogen evolution over the sample and to obtain a homogeneous growth inside the nanopores. Cobalt nanowires were grown in a mixed solution $^{18}(\mathrm{pH}=4.5)$ of $\mathrm{CoSO}_{4} \cdot 7 \mathrm{H}_{2} \mathrm{O}(252 \mathrm{~g} / \mathrm{L}), \mathrm{H}_{3} \mathrm{BO}_{3}$ $(50 \mathrm{~g} / \mathrm{L})$, and $\mathrm{NaCl}(7 \mathrm{~g} / \mathrm{L})$ at $25^{\circ} \mathrm{C}$ with a constant applied voltage of $-0.95 \mathrm{~V}$. A saturated calomel electrode was used as reference in potentiostatic mode and a Co film as counter electrode. By measuring the experimental development of the deposition current in the electrochemical cell, we interrupted the growth process at different times before the complete filling of the nanopores. This allows a reliable control of the average nanowires length. The crystallographic structure and morphology of the samples were investigated by 


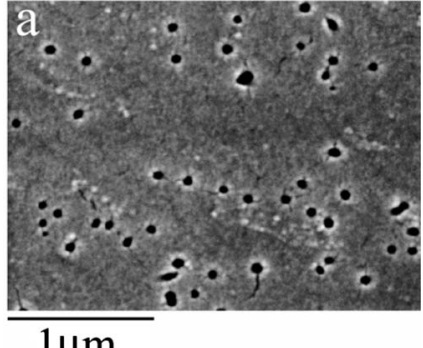

$1 \mu \mathrm{m}$

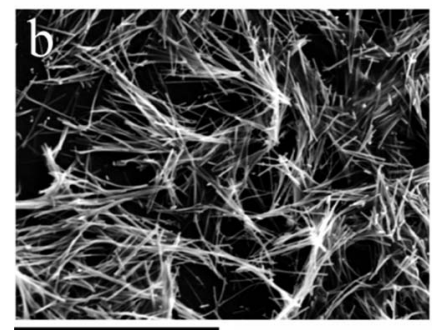

$10 \mu \mathrm{m}$

FIG. 1. SEM images of (a) a small area of a track-etched polycarbonate membrane and (b) Co nanowires after dissolution of the substrate. The images were recorded at $24 \mathrm{KV}$ and $43000 \times$ and $6000 \times$, respectively.

$\mathrm{X}$-ray diffraction (XRD) and scanning electron microscope (SEM). The field-dependent magnetization hysteresis of the nanowires was measured at room temperature in a LDJ Vibrating Sample Magnetometer with a external magnetic field of $1 \mathrm{~T}$ applied parallel $\left(H^{\|}\right)$and perpendicular $\left(H^{\perp}\right)$ to the nanowire symmetry axis. Temperature-dependent measurements were performed in a Quantum Design XL7 superconducting quantum interference device magnetometer in the (5-300) $\mathrm{K}$ range.

Figures 1(a) and 1(b) show the high-resolution SEM micrographs of the membrane surface and nanowires morphology, respectively. Both measurements were performed with a JEOL Scanning Microscope JSM-6400 working at $24 \mathrm{kV}$. Figure 1(a) corresponds to a polycarbonate membrane with $6 \mu \mathrm{m}$ thickness and $50 \mathrm{~nm}$ nominal pore diameter. Some defects are observed, probably due to stressing effects, as well as a low-ordering degree of nanopores. The pore density and pore diameter distributions can be estimated from this type of images, as it has been described in details elsewhere. ${ }^{19}$ The measured mean pore density of $(6.1 \pm 2.4) \times 10^{8}$ nanopores $\mathrm{cm}^{-2}$ is in good agreement with the value of $6 \times 10^{8}$ nanopores $\mathrm{cm}^{-2}$ given by the manufacturer. Figure 1(b) shows Co nanowires with $50 \mathrm{~nm}$ nominal diameter and $\sim 3 \mu \mathrm{m}$ length after dissolution of the polycarbonate membrane. While inside the membrane all nanowires are parallel to each other, after its dissolution they reorganize in different positions all over the sample area. In this way, the length of the nanowires can be measured more accurately and compared to the chronoamperometric measurements as explained in Ref. 19. Note that in the Fig. 1(a), the apparent deviations from a circular shape of the nanowires occurs only near the surface. This is due to the fabrication process of the polycarbonate membranes which typically leads to a conical opening of the nanopores which extends a few na-

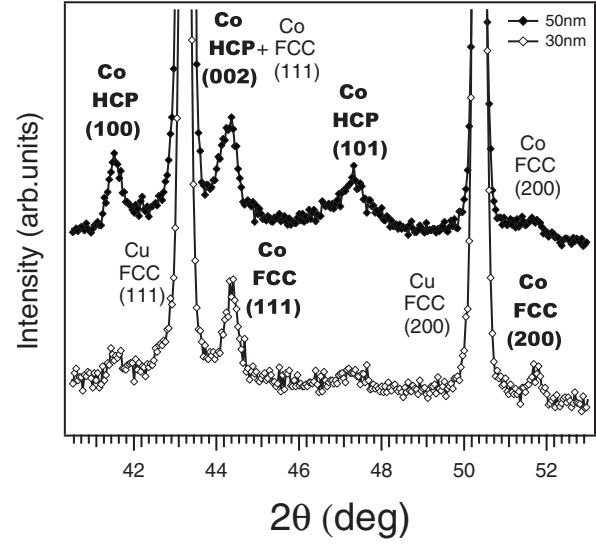

FIG. 2. Comparison of the x-ray diffraction patterns of electrodeposited Co nanowires with 30 and $50 \mathrm{~nm}$ nominal diameter.

nometers deep from the membrane surface. Therefore, we have taken particular care and ended the deposition process before the nanopores were completely filled with the magnetic material.

For the XRD measurements we have utilized a SIEMENS D-5000 diffractometer providing a $\mathrm{Cu} \mathrm{K}_{\alpha}$ radiation $(\lambda$ $=1.5418 \AA$ ) under working conditions of $40 \mathrm{kV}$ and $30 \mathrm{~mA}$. The measurements were done prior to dissolution of the polycarbonate membrane [as seen in Fig. 1(a)], when all nanowires are parallel to each other and the sputtered $\mathrm{Cu}$ layer on one side of the membrane was still present. Figure 2 shows the $\theta-2 \theta$ diffraction patterns of Co nanowires with $\sim 6 \mu \mathrm{m}$ length and nominal pore diameters of 30 and $50 \mathrm{~nm}$. Contributions from the $\mathrm{Cu}$ substrate appear as intense peaks corresponding to $\mathrm{Cu}-\mathrm{fcc}$ (111) and (200) orientations. The two diffractograms exhibit different crystalline structures as a function of the nanowire diameter. The diffraction pattern of nanowires with $30 \mathrm{~nm}$ diameter is dominated by a highly textured Co-fcc phase oriented along the [111] and [200] directions. The nanowires with $50 \mathrm{~nm}$ diameter exhibit, however, a more complicated diffraction pattern which indicates that a mixture of Co-hcp and Co-fcc phases is present. This suggests that Co nanowires are composed of hcp and fcc crystalline Co segments. ${ }^{20}$ We basically observe two new prominent structures corresponding to a Co-hcp phase oriented along the [100] and [101] directions. Their coexistence gives rise to an asymmetric peak appearing near the expected angular positions of the Co-hcp (002) and Co-fcc (111) phases. The double structure behind this peak cannot be resolved in this case, indicating that the degree of coexistence between the two crystalline phases is dominated by the Cohcp contribution.

In addition, other features, as the one corresponding to Co-fcc (200) phase, are barely distinguishable from the background signal. Since the final texture of the nanowires also depends on the plating procedure, it is still unclear why the amount of fcc phase increases with decreasing nanowire diameter. In recent studies, a coexistence of two different $\mathrm{Co}$ phases with different orientations for nanowires with $200 \mathrm{~nm}$ in diameter was also found. ${ }^{20} \mathrm{~A}$ transition to a Co-hcp crystalline structure was shown for nanowires with $65 \mathrm{~nm}$ in diameter and $\mathrm{a} \mathrm{pH}$ and diameter-dependent phase diagram 


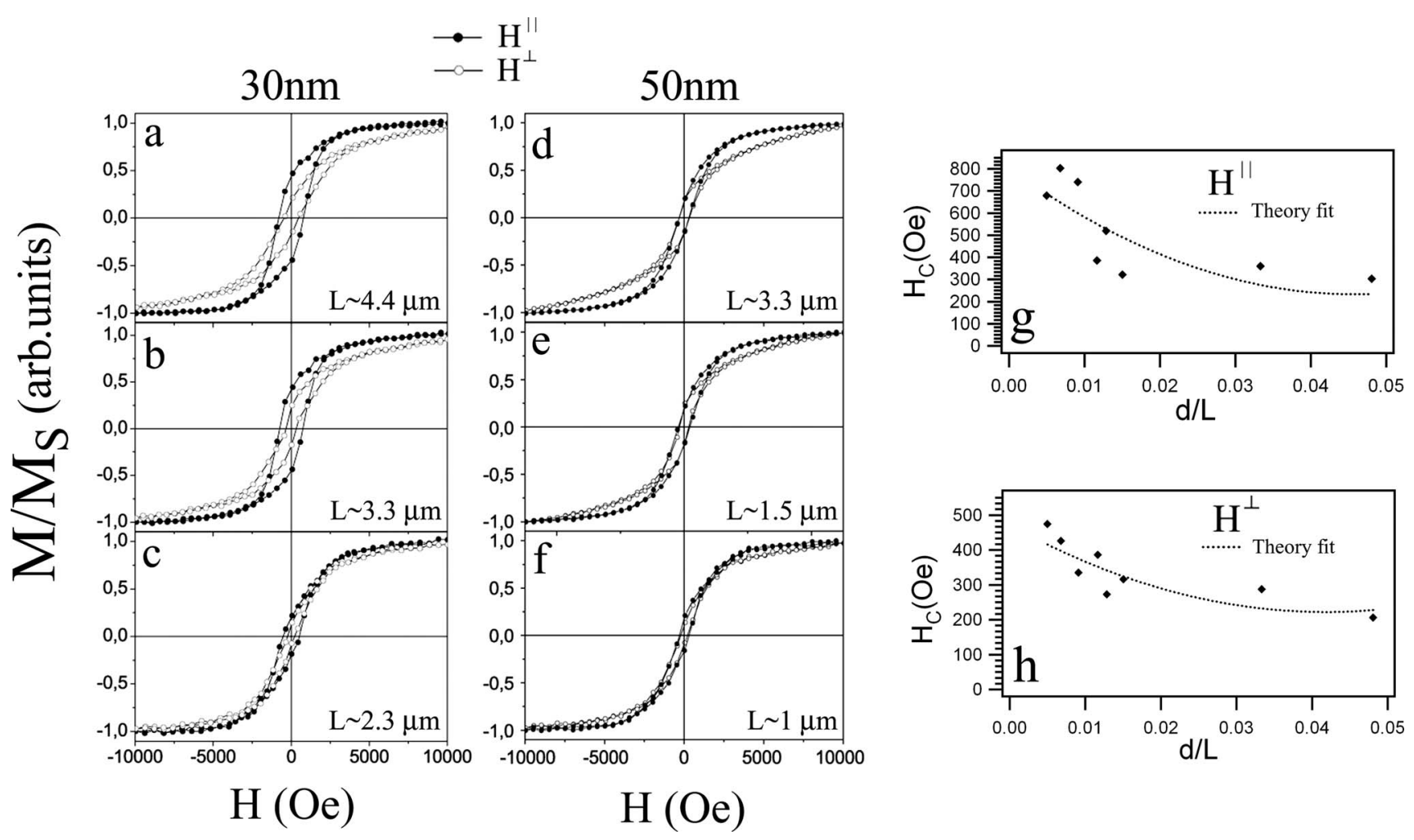

FIG. 3. Room-temperature field-dependent magnetization hysteresis of Co nanowires with different lengths and diameters. [(a)-(c)]: nanowires with $30 \mathrm{~nm}$ in diameter and lengths of $\sim 4.4, \sim 3.3, \sim 2.3 \mu \mathrm{m}$, respectively; [(d)-(f)]: nanowires with $50 \mathrm{~nm}$ in diameter and $\sim 3.3, \sim 1.5, \sim 1 \mu \mathrm{m}$ in length. $[(\mathrm{g})$ and $(\mathrm{h})]$ : general dependence of the coercive field as a function of the ratio $(d / L)$ of the nanowires when the magnetic field is applied parallel $\left(H^{\|}\right)$and perpendicular $\left(H^{\perp}\right)$ to the nanowire axis.

for electroplated Co nanowires was proposed. Our results give complementary information to the proposed phase diagram in Ref. 20, indicating that by further reduction in the nanowire diameter a second transition to a Co-fcc phase occurs for nanowire diameters of $\sim 30 \mathrm{~nm}$. Other investigations of Co nanoparticles ${ }^{21}$ show that the presence of a hcp phase or a fcc phase can be related to the particle diameter. In that case, the transition from hcp to fcc was ascribed to a size effect due to the lower surface energy of the fcc phase.

Figures 3(a)-3(f) show the resulting hysteresis loops measured for nanowire arrays with different diameters and lengths. Figures $3(\mathrm{~g})$ and $3(\mathrm{~h})$ show the extracted coercive field values and the corresponding theoretical fits (see below) as a function of the nanowire diameter-to-length ratio for both $\left(H^{\|}\right)$and $\left(H^{\perp}\right)$ externally applied fields, respectively. A better understanding of the observed dependence of the coercive field with the nanoscale dimensions observed in Figs. $3(\mathrm{~g})$ and $3(\mathrm{~h})$ can only be achieved by analyzing the effects of the different anisotropy contributions to the total anisotropy of the system, as it will be shown in the following. The first observation is a pronounced reduction in the coercivity and remanent magnetization values for nanowires with 50 $\mathrm{nm}$ in diameter [Figs. 3(d)-3(f)]. Besides, by comparing the axial and transverse hysteresis loops for each case, we observe how the effective magnetic anisotropy along the nanowire axis increases with increasing length for both diameters. The longer the nanowires, the larger the remanent magnetization and coercive field values for an applied field parallel to the nanowire axis $\left(H^{\|}\right)$. This effect appears more pro- nounced for nanowires with $30 \mathrm{~nm}$ in diameter [Figs. 3(a)-3(c)].

We argue below that the effective magnetic anisotropy is mainly oriented along the nanowire axis in all cases, and that the shape anisotropy of the nanowire arrays is playing a more dominant role than the dipolar interaction for our particular system. The shape anisotropy of an infinitely long wire would result in a squared hysteresis loop exhibiting a finite coercive field for an applied field along the wire $H^{\|}$and zero coercive field for a perpendicular applied field to the wire $H^{\perp}$. Within the Stoner-Wohlfarth model, a particular case is the so-called prolate spheroid geometry of magnetic objects. If the ellipsoid is uniformly magnetized, the magnetic field inside the ellipsoid depends directly on the demagnetization tensor $\left(\vec{H}_{i n} \sim-N \vec{M}\right)$. Since the trace of the tensor $N$ must be 1 , for a limiting case of a infinitely long cylinder in which the shape anisotropy plays the dominant role one would obtain $N_{x}=N_{y}=1 / 2$ and $N_{z}=0$. In a general case, the demagnetizing factor along the long axis of the ellipsoid (or, by analogy, along the cylinder) depends on second-order terms of the type $(d / L)^{2}$ and it approaches 0 when $L \rightarrow \infty$. Since $\vec{H}_{c} \sim \vec{H}_{a}-N \vec{M}$, this leads to the following expectation for the coercive field: $\vec{H}_{c} \rightarrow \vec{H}_{a}$ when $N \rightarrow 0$, where $H_{a}$ is the intrinsic anisotropy field. Therefore, a system with finite dimensions which is dominated by shape anisotropy $(d \ll L)$ exhibits a finite $N_{z}\left(N_{z} \neq 0\right)$. Our observations in Fig. 3, where coercive field values for $H^{\|}$are larger than the for $H^{\perp}$, are fully consistent with shape anisotropy origin of the enhanced coercivity as discussed qualitatively within the Stoner- 
Wohlfarth model. Nevertheless, other contributions to the effective anisotropy of the whole nanowire array, other than the shape induced anisotropy, may influence the observed magnetic behavior: dipolar interactions among nanowires, magnetocrystalline anisotropy, and magnetoelastic anisotropy. ${ }^{22}$ As it has been shown for nanowires grown in alumina membranes, the former certainly depends strongly on the interwire distances and typically manifests through a decreasing coercive field along $H^{\|}$with an increasing ratio of nanowire diameter to interwire spacing. ${ }^{23}$ Although the use of randomly distributed array of nanopores which is characteristic of the polycarbonate membranes does not allow an exhaustive control of the interwire distances, an average value of $\sim 500 \mathrm{~nm}$ can be deduced, which is one order of magnitude larger than the typical nanowire diameter. Therefore, for our case the dipolar interactions have a minor effect for sufficiently long nanowires. A reminiscent influence of the dipolar interaction on the magnetic behavior would manifest through a small contribution to the reduced remanent magnetization for shorter wires. Further discussion about the role of the magnetocrystalline anisotropy is provided below by interpreting the measured hysteresis loops in combination with the different orientations of the crystalline structure observed in the x-ray diffraction patterns of Fig. 2.

For nanowires which are $30 \mathrm{~nm}$ in diameter, the [111] direction of the Co-fcc phase is mainly oriented along the axis of the nanowires, with the basal (111) planes being parallel to the membrane plane. For nanowires which are $50 \mathrm{~nm}$ in diameter, the Co-hcp [100] and [002] directions are oriented perpendicular and parallel to the nanowire axis, respectively. Moreover, the $c$ axis of the hcp (101) phase makes an angle of $32^{\circ}$ with respect to the nanowire axis. As a result, the effective crystalline alignment resulting from the different Co-hcp orientations observed in the diffraction pattern would lead to a reinforcement of the total magnetocrystalline anisotropy of the system along the $H^{\perp}$ direction. For a Cofcc (111) phase oriented along the nanowire axis, the magnetocrystalline anisotropy energy density $K_{1}=6.3$ $\times 10^{5} \mathrm{erg} / \mathrm{cm}^{3}$ is almost one order of magnitude smaller than the shape anisotropy energy density $K_{S}=\pi \cdot M_{S}^{2}=6.0$ $\times 10^{6} \mathrm{erg} / \mathrm{cm}^{3}$, whereas for a Co-hcp structure, they are of the same order of magnitude $\left(K_{1}=5 \times 10^{6} \mathrm{erg} / \mathrm{cm}^{3} \sim K_{S}\right.$ $\left.=\pi \cdot M_{S}^{2}=6.0 \times 10^{6} \mathrm{erg} / \mathrm{cm}^{3}\right) .{ }^{24}$ Hence, for nanowires with $50 \mathrm{~nm}$ diameter, we can expect a more pronounced interplay of both magnetocrystalline and shape anisotropies that results in a slightly weaker effective anisotropy along the nanowire axis. This leads to a further reduction in the coercive field and remanent magnetization for $H^{\|}$as compared to the $30 \mathrm{~nm}$ wires. In this respect, the reduced coercivity for larger diameters depends not only on the geometrical properties, i.e., the nanowire diameter dependence of the coercive field, ${ }^{25}$ but also on the partial influence of the crystalline orientation of the Co-hcp phase, i.e., on magnetocrystalline anisotropy.

As to further investigate the validity of the aforementioned effects, i.e., the interplay between magnetocrystalline and shape anisotropies, we present in Fig. 4 temperaturedependent measurements for nanowires with 30 and $50 \mathrm{~nm}$ diameter and $\sim 4$ and $\sim 3 \mu \mathrm{m}$ in length, respectively. Such type of measurements allow us to determine if other contri- butions, particularly the ones arising from magnetoelastic effects may influence the magnetic behavior of the nanowires system at room temperature. Figures 4(a)-4(f) show the resulting hysteresis loops measured for both systems of nanowire arrays at different temperatures. Figures $4(\mathrm{~g})$ and $4(\mathrm{~h})$ show the extracted coercive field $\left(H_{c}\right)$ and irreversible switching field $\left(H_{i r r}\right)$ values as a function of temperature for both parallel $\left(H^{\|}\right)$and perpendicular $\left(H^{\perp}\right)$ applied magnetic fields. $H_{i r r}$ is the field where the magnetization changes irreversibly (i.e., where the hysteresis opens). In Figs. 4(a)-4(f) we do not observe significant changes in the hysteresis loops when the temperature decreases down to $5 \mathrm{~K}$. The main observation is a quasimonotonic decrease in $H_{c}$ and $H_{i r r}$ with increasing temperature, as summarized in Figs. 4(g) and 4(h). This effect which is observed for both applied field directions, appears to be less pronounced in the case of the $H_{c}$ values extracted for $H^{\perp}$. As shown in Figs. 4(g) and 4(h), we performed linear fits to the experimental data from which we estimate the relative changes in the angle of orientation of the anisotropy axis $(\Theta), H_{c}$ and $H_{\text {irr }}$. We obtain $\Theta \sim 35^{\circ}$ and a partial reorientation of $\sim 6^{\circ}$ toward the nanowire axis when the temperature is decreased. For $H^{\|}$, the changes in $H_{c}$ with respect to its low temperature value are $\sim 23 \%$ and $\sim 38 \%$ for nanowires with 30 and $50 \mathrm{~nm}$ in diameter, respectively. For an applied field $H^{\perp}$, these changes are $\sim 15 \%$ and $\sim 24 \%$, respectively. The relative increase in $H_{\text {irr }}$ is $\sim 47 \%$ in all cases. Note that in general, (i) this effect is more pronounced in the $H_{i r r}$ values and that (ii) $H_{c}$ is larger for $H^{\|}$ than for $H^{\perp}$, whereas $H_{i r r}$ is smaller for $H^{\|}$than for $H^{\perp}$. This differences are expected from the Stoner-Wohlfarth theory, and they can be qualitatively explained by considering the calculated azimuthal dependence of both $H_{i r r}$ and $H_{c}$ for a magnetic system with uniaxial anisotropy. ${ }^{26}$ Our results indicate that only a moderate increase in the effective anisotropy of the nanowire system along the nanowire axis occurs at low temperatures, resulting in a rather small reorientation of the anisotropy axis along the nanowires. Previous studies of $\mathrm{Ni}$ nanowires showed that magnetoelastic effects can have profound influences on the magnetic properties of the arrays, ${ }^{27,28}$ typically leading to a reorientation of the magnetic easy axis of the nanowire system from the parallel to perpendicular configuration with decreasing temperature. At low temperatures, the nanowires are under stress due to the large mismatch between the thermal expansion coefficients of $\mathrm{Co}\left(\alpha_{\mathrm{Co}} \sim 13 \times 10^{-6} \mathrm{~K}^{-1}\right)$ and the polycarbonate membrane $\left(\alpha_{\text {Polyc }} \sim 67 \times 10^{-6} \mathrm{~K}^{-1}\right)$. Since $\alpha_{\text {Polyc }}>\alpha_{\text {Co }}$, the polycarbonate template tends to contract more than the Co during cooling, resulting in a transverse compressive force which acts perpendicular to the nanowire axis and leads to an expansion of the nanowires along their axis. The axial strain produced in this process can be calculated as $\epsilon_{\|}=$ $-\nu \Delta \mathrm{T}\left(\alpha_{\mathrm{Co}}-\alpha_{\text {Polyc }}\right)$, where $\nu$ is the Poisson's ratio of $\mathrm{Co}$ $\left(\nu_{\mathrm{Co}}=0.31\right)$ and $\Delta T=295 \mathrm{~K}$ when the temperature varies from 300 to $5 \mathrm{~K}$. This is equivalent to an axial tensile stress of $\sigma_{\|}=E_{\mathrm{Co}_{0}} \epsilon_{\|} \sim 1.03 \mathrm{GPa}$, where $E_{\mathrm{Co}} \sim 209 \mathrm{GPa}$ is the Young's modulus of Co. Considering that the saturation magnetostriction constant of $\mathrm{Co}$ is negative $\left(\lambda_{s}^{\mathrm{Co}} \sim-55\right.$ $\left.\times 10^{-6}\right),{ }^{29,30}$ such an axial effective tensile stress will decrease the effective anisotropy along the nanowire axis and, therefore the $H_{c}$ and $H_{i r r}$ values, in contrast to our observa- 

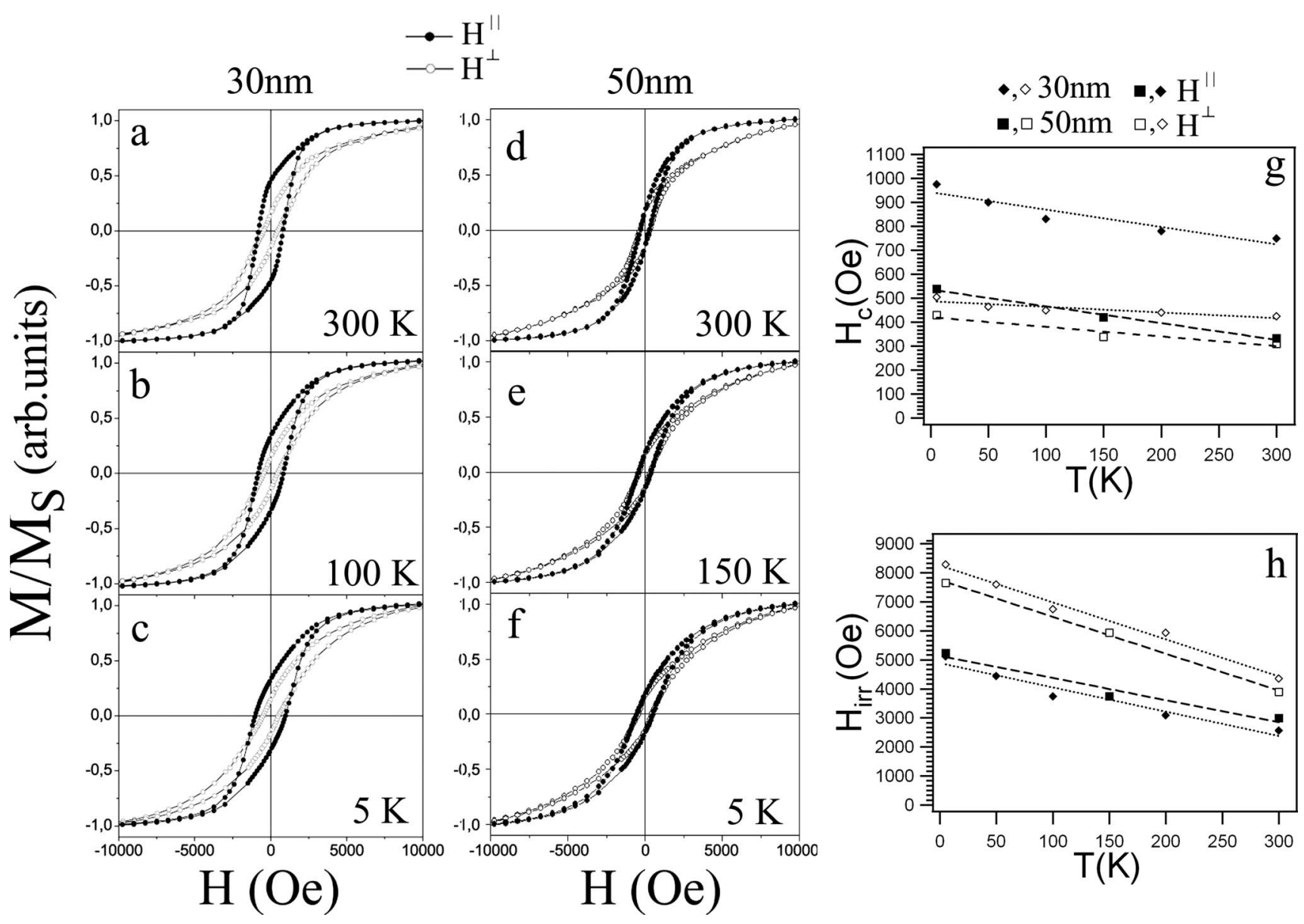

FIG. 4. Field-dependent magnetization hysteresis of Co nanowires at different temperatures. [(a)-(c)]: nanowires with $30 \mathrm{~nm}$ in diameter and $\sim 4 \mu \mathrm{m}$ in length; [(d)-(f)]: nanowires with $50 \mathrm{~nm}$ in diameter and $\sim 3 \mu \mathrm{m}$ in length. [(g) and (h)]: general dependence of the coercive and irreversible fields as a function of temperature for both parallel $\left(H^{\|}\right)$and perpendicular $\left(H^{\perp}\right)$ applied magnetic fields. Dotted and dashed lines result from linear fits of the experimental data for nanowires with nominal diameters of 30 and $50 \mathrm{~nm}$, respectively.

tions. In some instances, when the magnetoelastic effects are very strong, ${ }^{30}$ the interplay between magnetoelastic and shape anisotropies may lead to a reorientation of the anisotropy axis at certain crossover temperature below which $H_{c}^{\perp}>H_{c}^{\|}$. The maximum contribution of the transverse magnetoelastic anisotropy energy density can be estimated as $K_{m e}=3 \lambda_{s}^{\mathrm{Co}} \sigma_{\|} / 2 \sim-8.4 \times 10^{5} \mathrm{erg} / \mathrm{cm}^{3}$ which is one order of magnitude smaller than the shape anisotropy energy density $K_{S}=6.0 \times 10^{6} \mathrm{erg} / \mathrm{cm}^{3}$. Therefore, the effective magnetoelastic anisotropy is not playing a dominant role in the remagnetization processes of individual Co nanowires. Note that the calculated value of $K_{m e}$ represents an upper limit since we assume perfect adhesion of the nanowires to the pore walls. In this simplified calculation, we have neglected (i) the weak dependence of the $E_{\mathrm{Co}}$ with the nanowire length, (ii) the losses in the compression energy due to the large mismatch between the Young's modulus of $\mathrm{Co}$ and the polycarbonate membrane $\left(E_{\text {Polyc }} \sim 2 \mathrm{GPa} \ll E_{\mathrm{Co}}\right)$, (iii) nonlinear effects in the temperature-dependent expansion coefficients, and (iv) other second-order effects in the temperature dependence of the elastic properties. We attribute the observed effects to a preferential increase of the effective magnetocrystalline anisotropy with decreasing temperature. While cooling down to $5 \mathrm{~K}$, the $H_{c}$ and $H_{\text {irr }}$ are increasing due to the thermal energy which acts against the anisotropy energy. At low temperatures, the relative changes in $H_{c}$ and $H_{i r r}$ will depend on the size and temperature-dependent behavior of the magnetocrystalline anisotropy, and therefore on the orientation of the different crystalline structures appearing in the $\mathrm{x}$-ray diffraction patterns of Fig. 2. For nanowires with $30 \mathrm{~nm}$ in diameter, the relative increase in the magnetocrystalline anisotropy along the nanowire axis is $\sim 27 \%$, whereas $\sim 40 \%$ for nanowires with $50 \mathrm{~nm}$ in diameter. This indicates that the dominant contributions in the diffraction patterns are the Co-hcp (002) and Co-fcc (111) phases for nanowires with 50 and $30 \mathrm{~nm}$ in diameter, respectively.

In order to gain more insight on the interplay between both magnetocrystalline and shape anisotropies, we would like to come back to the results shown in Figs. 3(g) and 3(h) and provide further theoretical analysis of the observed room-temperature dependence of the coercive field as a function of the diameter-to-length ratio $(d / L)$ of the nanowires. Such type of analysis gives more information about the origin of the dominant anisotropy contributions to the total magnetic anisotropy of the system and, particularly, how strongly the remagnetization process of the nanowires depend on the shape anisotropy, i.e., on the nanowire dimensions. Based on the aforementioned argumentations, our the- 
oretical analysis does not consider contributions to the effective anisotropy of the nanowire system arising from magnetoelastic effects and dipolar interactions among nanowires. As previously mentioned, the results of Figs. 3(g) and 3 (h) are presented together with a fit function $H_{c}^{\|, \perp}$ $=f^{\|, \perp}(d / L)$ which explicitly shows a size-dependent behavior as it will be deduced in the following. The physical origin of this dependence are demagnetizing fields, ${ }^{31,32}$ which give rise to the shape anisotropy of the nanowires and, therefore, it allow us to check the validity of a simple model in which the shape anisotropy is the dominant contribution to the observed size-dependent coercive field behavior. The relation between the demagnetizing factors $N^{\|}$or $N^{\perp}$ and the structure dimensions can be expressed in terms of the hypergeometric Gauss function ${ }_{2} \mathrm{~F}_{1} \cdot{ }^{33}$ In the limit in which $d \ll L$ and taking into account that any given finite circular cylinder with diameter $d$ and length $L$ possesses rotational symmetry about its geometrical axis, for $\kappa=d / L, N^{\|}$can be written as ${ }^{34}$

$$
N^{\|}=\frac{4}{3 \pi} \kappa-8 \kappa^{2}+O\left(\kappa^{4}\right),
$$

which represents a second-order dependence of the demagnetizing factor on the diameter-to-length ratio of the nanowires. We can consider that the expressions for both coercive fields $H_{c}^{\|}$and $H_{c}^{\perp}$ are given by ${ }^{26}$

$$
H_{c}^{\|}=\frac{2 K_{e f f}}{\mu_{0} M_{s}}|\cos \Theta|, \quad H_{c}^{\perp}=\frac{2 K_{e f f}}{\mu_{0} M_{s}}|\sin \Theta|
$$

with $K_{\text {eff }}$ being the effective anisotropy constant of the ferromagnet, $M_{s}$ the saturation magnetization, and $\Theta$ the angle between the applied field and the anisotropy axis of the system. Considering that $N^{\|}-N^{\perp}=\left(3 N^{\|}-1\right) / 2$, a widely used phenomenological expression for $K_{\text {eff }}$ is given by

$$
\begin{gathered}
\epsilon_{k} K_{M}-\frac{\left(1-3 N^{\|}\right) \mu_{0} M_{s}^{2}}{4} \quad \text { if } d<d_{c}, \\
\epsilon_{k} K_{M}+\frac{c\left(N^{\|}\right) A}{(d / 2)^{2}}-\frac{N^{\|} \mu_{0} M_{s}^{2}}{2} \quad \text { if } d>d_{c},
\end{gathered}
$$

where $\epsilon_{k}$ is the real-structure-dependent Kronmüller parameter, ${ }^{35} K_{M}$ is the effective magnetocrystalline anisotropy constant, $d_{c}$ is the critical coherence diameter, and $A$ is the exchange stiffness constant $\left(A=30 \times 10^{-12} \mathrm{~J} / \mathrm{m}\right.$ for Co $)$. If $d<d_{c}$, then the dominant exchange interaction yields coherent (uniform) rotation [Eq. (3)], whereas if $d>d_{c}$ magnetostatic interactions give rise to curling reversal [Eq. (4)]. The critical diameter, defined as $d_{c}=3.68 \sqrt{A / \pi M_{s}^{2}}$ yields to $d_{c} \sim 32 \mathrm{~nm}$ for Co. The second term on the right hand of Eq. (3) represents the shape anisotropy contribution to the effective anisotropy of the system while in Eq. (4) the exchange term partly compensates for the absence of shape anisotropy in a proper sense. Considering that $c\left(N^{\|}\right)=\left(M_{s} d_{c}\right)^{2} N^{\perp}$ and by substituting Eq. (3) and (4) into Eq. (2) and then introducing Eq. (1), after some manipulation the coercive field becomes
TABLE I. Values of the fitted parameters $\alpha^{\|, \perp}, \beta^{\|, \perp}$, and $\gamma^{\|, \perp}$ for both applied field directions $H^{\|}$and $H^{\perp}$, respectively.

\begin{tabular}{cccc}
\hline \hline & $\begin{array}{c}\alpha^{\|, \perp} \\
(\mathrm{Oe})\end{array}$ & $\begin{array}{c}\beta^{\|, \perp} \\
(\mathrm{Oe})\end{array}$ & $\begin{array}{c}\gamma^{\|, \perp} \\
(\mathrm{Oe})\end{array}$ \\
\hline$H^{\|}$ & $805 \pm 108$ & $(2.5 \pm 0.6) \times 10^{4}$ & $(2.7 \pm 1.3) \times 10^{5}$ \\
$H^{\perp}$ & $472 \pm 60$ & $(1.2 \pm 0.3) \times 10^{4}$ & $(1.4 \pm 0.6) \times 10^{5}$ \\
\hline \hline
\end{tabular}

$$
H_{c}^{\|, \perp}=\alpha^{\|, \perp}-\beta^{\|, \perp}\left(\frac{d}{L}\right)+\gamma^{\|, \perp}\left(\frac{d}{L}\right)^{2} .
$$

The relation between the physical constants and the fitted parameters $\alpha^{\|, \perp}, \beta^{\|, \perp}$, and $\gamma^{\|, \perp}$ can be written as follows:

$$
\frac{\left(\alpha^{\|}, \beta^{\|}, \gamma^{\|}\right)}{|\cos \Theta|}=\frac{\left(\alpha^{\perp}, \beta^{\perp}, \gamma^{\perp}\right)}{|\sin \Theta|}=(A, B, C)
$$

with $A, B$, and $C$ given by

$$
\begin{gathered}
A=\alpha\left(\frac{2 \epsilon_{k} K_{M}}{\mu_{0} M_{s}}+\frac{g M_{s}}{2}\right), \quad B=\frac{4 \beta M_{s}}{3 \pi}\left(1+\frac{g}{2}\right), \\
C=8 \gamma M_{s}\left(1+\frac{g}{2}\right) \quad \text { with } g=\left(\frac{d_{c}}{d}\right)^{2},
\end{gathered}
$$

where $g=1$ for coherent rotation. We have introduced the parameters $\alpha, \beta$, and $\gamma$ in order to account for quantitative deviations from the situation in which the magnetization reversal processes are driven by coherent-rotation or curling mechanisms. Certainly, since the diameters of the fabricated nanowires are near to the limit for coherent rotation it is not straightforward to deduce which magnetization reversal process is the preferred mechanism. For curling processes and within the present approximation, we assume that $d_{c}$ is not decreasing strongly with increasing $d / L$. That means that $0.20 \leq g / 2 \leq 0.56$ for nanowires with 50 and $30 \mathrm{~nm}$, respectively. The results of the fit using the function, Eq. (5), show qualitative agreement to the experiment and are represented in Figs. 3(g) and 3(h). The fitted parameters are given in Table I. From the experimental data obtained at maximum and minimum $d / L$ values we estimate a nearly constant angle of rotation respect to the nanowire axis of $\Theta \sim 32^{\circ}$, and its variation is below $8^{\circ}$ for a decreasing structure size. In agreement to it, the theoretical fit gives $\Theta=(28 \pm 3)^{\circ}$. Therefore, we suggest that the observed changes in the coercive field are mainly due to an increase in the effective magnetic anisotropy $K_{\text {eff }}$ of the system, rather than due to a rotation of the anisotropy axis toward the nanowire axis. Considering $\epsilon_{k}=1$ and introducing $M_{s}(\mathrm{Co}) \sim 1424$ Oe, for $\alpha=1$ and $g$ $=1$ we estimated $K_{M} \sim(1.8 \pm 0.4) \times 10^{6} \mathrm{erg} / \mathrm{cm}^{3}$, in agreement with the average value of $\sim 2.8 \times 10^{6} \mathrm{erg} / \mathrm{cm}^{3}$ obtained from the magnetocrystalline anisotropy energy densities of Co-fcc and hcp crystalline structures. The estimated value of $K_{M}$ indicates that the changes in the magnetocrystalline anisotropy density of the system are mostly due to a mixture of both fcc and hcp crystal orientations, in agreement with the x-ray diffraction patterns of Fig. 2. For $g=1$, the deviations from the first and second order terms yield to 
$\beta \sim(30 \pm 8)$ and $\gamma \sim(18 \pm 9)$, whereas for $0.20 \leq g / 2$ $\leq 0.56, \beta \sim(34 \pm 10)$ and $\gamma \sim(20 \pm 11)$. Although a qualitative agreement is reached in the general dependence of the coercive field with $d / L$, this deviations indicate that the Stoner-Wohlfarth approximation is overestimating the coercive field values by a factor of $\sim 3$ for both applied directions. In this respect, it was shown that at the wire ends a butterfly type arrangement of the magnetization exists which reduces the switching field considerably. ${ }^{36}$ Micromagnetic simulations for $\mathrm{Ni}$ nanowires of $40 \mathrm{~nm}$ diameter, also showed that the nucleation field can be typically reduced by a factor of $2 .{ }^{37}$ In addition, in the case of an array of nanowires, collective demagnetization modes have to be taken into account which lead to a further decrease in the coercive field. The reduced squareness observed in the hysteresis loops of Fig. 3 might result from the existence of polycrystalline and surface-related structural imperfections along the nanowires, leading to a magnetic localization of the reversal modes. ${ }^{38}$ The coherent-rotation and curling modes are delocalized, which means that the spatial variations in the magnetization along the nanowire are small. However, local variations in the magnetization cost some exchange energy but they may be energetically favorable from the point of view of a local anisotropy, $K_{e f f}(r)$. This competition leads not only to a reduction in the nucleation field but also to a localization of the magnetization reversal modes and to the formation of magnetic domains along the wires. Further understanding of the real-space character of the magnetization mechanisms can be achieved by considering cooperative effects between coherent-rotation and curling modes. ${ }^{39}$ Actu- ally this type of processes are of great importance in advanced technology because they lead to the formation of interactive magnetic domains which may improve the thermal stability of the samples or reduce the storage densities in the case of extended magnets or ultrathin films.

In conclusion, we have shown that the magnetic properties of the fabricated Co nanowire arrays are dominated by the shape anisotropy contributions more than the magnetocrystalline anisotropy, magnetoelastic effects, and dipolar interactions among nanowires. A partial influence of the different crystallographic orientations on the magnetic hysteresis loops at room temperature is deduced by analyzing the $\mathrm{x}$-ray diffraction patterns. Low-temperature measurements indicate that the magnetoelastic anisotropy is not playing a dominant role in the remagnetization process of the nanowires. The changes are attributed to a temperature-dependent behavior of the magnetocrystalline anisotropy. We have developed a phenomenological model on the basis of the StonerWohlfarth theory which reproduces the dependence of the coercive field on the nanowire diameter-to-length aspect ratio at room temperature. The increase in coercive field as $d / L$ decreases is attributed to an enhancement of the effective magnetic anisotropy of the system for a nearly constant angle of orientation respect to the applied magnetic field. The quantitative analysis of the coercive field within the proposed model suggests additional contributions to remagnetization mechanisms, discussed in terms of localization and cooperative effects of the magnetization reversal modes due to polycrystalline and surface-related structural imperfections in the nanowires.

\footnotetext{
*sbarriga@bessy.de

${ }^{1}$ P. Grünberg, R. Schreiber, Y. Pang, M. B. Brodsky, and H. Sowers, Phys. Rev. Lett. 57, 2442 (1986).

${ }^{2}$ M. N. Baibich, J. M. Broto, A. Fert, F. Nguyen Van Dau, F. Petroff, P. Etienne, G. Creuzet, A. Friederich, and J. Chazelas, Phys. Rev. Lett. 61, 2472 (1988).

${ }^{3}$ G. Binasch, P. Grünberg, F. Saurenbach, and W. Zinn, Phys. Rev. B 39, 4828 (1989).

${ }^{4}$ S. Morup, D. E. Madsen, C. Frandsen, C. R. H. Bahl, and M. F. Hansen, J. Phys.: Condens. Matter 19, 213202 (2007).

${ }^{5}$ E. L. Salabas, A. Rumplecker, F. Kleitz, F. Radu, and F. Schüth, Nano Lett. 6, 2977 (2006).

${ }^{6}$ J. I. Martin, J. Nogues, K. Liu, J. L. Vicent, and I. K. Schuller, J. Magn. Magn. Mater. 256, 449 (2003).

${ }^{7}$ S. S. P. Parkin, M. Hayashi, and L. Thomas, Science 320, 190 (2008).

${ }^{8}$ H. J. Richter, J. Phys. D 40, R149 (2007).

${ }^{9}$ W. Wernsdorfer, D. Mailly, and A. Benoit, J. Appl. Phys. 87, 5094 (2000).

${ }^{10}$ J. Bachmann, J. Escrig, K. Pitzschel, J. M. Montero Moreno, J. Jing, D. Görlitz, D. Altbir, and K. Nielsch, J. Appl. Phys. 105, 07B521 (2009).

${ }^{11}$ M. Bolte, R. Eiselt, G. Meier, D-H. Kim, and P. Fischer, J. Appl. Phys. 99, 08H301 (2006).

${ }^{12}$ M. J. Benitez, O. Petracic, E. L. Salabas, F. Radu, H. Tüysüz, F.
}

Schüth, and H. Zabel, Phys. Rev. Lett. 101, 097206 (2008).

${ }^{13}$ J. F. Smyth, S. Schultz, D. Kern, and H. Schmid, J. Appl. Phys. 63, 4237 (1988).

${ }^{14}$ M. Shiraki, Y. Wakui, T. Tokushima, and N. Tsuya, IEEE Trans. Magn. 21, 1465 (1985).

${ }^{15}$ M. Darques, A. Encinas, L. Vila, and L. Piraux, J. Phys. D: Appl. Phys. 37, 1411 (2004).

${ }^{16}$ V. Ganesh, D. Vijayaraghavan, and V. Lakshminarayanan, Appl. Surf. Sci. 240, 286 (2005)

${ }^{17}$ H. Masuda and K. Fukuda, Science 268, 1466 (1995).

${ }^{18}$ T. M. Whitney, J. S. Jiang, P. C. Searson, and C. L. Chien, Science 261, 1316 (1993).

${ }^{19}$ J. Sánchez-Barriga, M. Lucas, G. Rivero, P. Marin, and A. Hernando, J. Magn. Magn. Mater. 312, 99 (2007).

${ }^{20}$ Zuxin Ye, Haidong Liu, Zhiping Luo, Han-Gil Lee, Wenhao Wu, D. G. Naugle, and I. Lyuksyutov, J. Appl. Phys. 105, 07 E126 (2009).

${ }^{21}$ O. Kitakami, H. Sato, Y. Shimada, F. Sato, and M. Tanaka, Phys. Rev. B 56, 13849 (1997).

${ }^{22}$ J. Escrig, D. Altbir, M. Jaafar, D. Navas, A. Asenjo, and M. Vázquez, Phys. Rev. B 75, 184429 (2007).

${ }^{23}$ M. Vázquez, K. Pirota, J. Torrejón, D. Navas, and M. Hernández-Vélez, J. Magn. Magn. Mater. 294, 174 (2005).

${ }^{24}$ F. Li, T. Wang, L. Ren, and J. Sun, J. Phys.: Condens. Matter 16, 8053 (2004). 
${ }^{25}$ J.-H. Gao, D.-L. Sun, Q.-F. Zhan, W. He, and Z.-H. Cheng, Phys. Rev. B 75, 064421 (2007).

${ }^{26}$ F. Radu and H. Zabel, Springer Tracts Mod. Phys. 227, 97 (2008).

${ }^{27}$ S. Dubois, J. Colin, J. L. Duvail, and L. Piraux, Phys. Rev. B 61, 14315 (2000).

${ }^{28}$ A. Kumar, S. Fähler, H. Schlörb, K. Leistner, and L. Schultz, Phys. Rev. B 73, 064421 (2006).

${ }^{29}$ O. Kazakova, B. Daly, and J. D. Holmes, Phys. Rev. B 74, 184413 (2006).

${ }^{30}$ K. R. Pirota, E. L. Silva, D. Zanchet, D. Navas, M. Vázquez, M. Hernández-Vélez, and M. Knobel, Phys. Rev. B 76, 233410 (2007).
${ }^{31}$ M. Beleggia, M. De Graef, Y. T. Millev, D. A. Goode, and G. Rowlands, J. Phys. D 38, 3333 (2005).

${ }^{32}$ B. Leven and G. Dumpich, Phys. Rev. B 71, 064411 (2005).

${ }^{33}$ Y. T. Millev, E. Vedmedenko, and H. P. Oepen, J. Phys. D 36, 2945 (2003).

${ }^{34}$ M. Beleggia, M. De Graef, and Y. T. Millev, J. Phys. D 39, 891 (2006).

${ }^{35}$ H. Kronmüller, Phys. Status Solidi B 144, 385 (1987).

${ }^{36}$ A. Holz, Phys. Status Solidi B 26, 751 (1968).

${ }^{37}$ H. Suhl and H. N. Bertram, J. Appl. Phys. 82, 6128 (1997).

${ }^{38}$ M. Zheng, R. Skomski, Y. Liu, and D. J. Sellmyer, J. Phys.: Condens. Matter 12, L497 (2000).

${ }^{39}$ R. Skomski and D. J. Sellmyer, J. Appl. Phys. 89, 7263 (2001). 